\title{
Prix Lucie-Samson-Turcotte, Lauréat 2013
}

\section{(2) OpenEdition \\ Journals}

Édition électronique

URL : http://journals.openedition.org/ere/856

DOI : $10.4000 /$ ere.856

ISSN : 2561-2271

Éditeur

Centr'ERE

Référence électronique

"Prix Lucie-Samson-Turcotte, Lauréat 2013 », Éducation relative à l'environnement [En ligne], Volume

11 | 2014, mis en ligne le 20 décembre 2013, consulté le 24 septembre 2020. URL : http://

journals.openedition.org/ere/856 ; DOI : https://doi.org/10.4000/ere.856 


\section{Prix Lucie-Samson-Turcotte, Lauréat 2013}

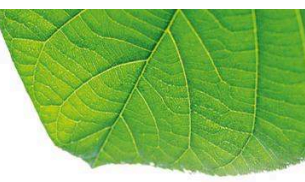

Concours 2013

Pour honorer la mémoire de la professeure Lucie Samson-Turcotte qui a marqué le domaine de l'éducation relative à l'environnement au Québec, la Faculté des sciences de l'éducation de l'Université Laval a créé à l'automne 1992, un prix visant à récompenser les mémoires de maîtrise et les thèses de doctorat de qualité qui contribuent de manière remarquable à l'avancement de l'éducation relative à l'environnement. En 2013, c'est dans le cadre de l'École d'été en Éducation à l'environnement et développement durable qui s'est tenue pour une quatrième année consécutive à l'Université Laval que ce prix a pu être remis (http://www.fse.ulaval.ca/ecole-ete/ecole-1).

L'année 2013 aura été particulièrement féconde puisque ce sont deux thèses de doctorat et un mémoire de maitrise qui ont pu être primés. Ces trois productions de grande qualité témoignent toutes de l'engagement de leur auteur dans un processus de recherche de grande envergure en éducation relative à l'environnement, alliant une théorisation importante et une connaissance riche du champ de recherche en question, à une étude empirique originale et d'envergure (menée dans plusieurs contextes dans le cas des thèses de doctorat), ce qui éclaire les théorisations proposées de positions d'acteurs auxquels on donne visiblement une grande importante et une belle crédibilité. Il faut ajouter que chacune de ces thèses, tout comme le mémoire de maîtrise qui a été primé, sont très bien écrits, ce qui permet une lecture tout à fait agréable et intéressante. 
3 Le prix Lucie-Samson-Turcotte 2013 pour une thèse de doctorat a ainsi été décerné à Madame Nayla Naoufal pour sa thèse de doctorat intitulée «La contribution de l'éducation relative à l'environnement à la construction d'une dynamique de paix ».

4 Les membres du jury ont tenu à en souligner le caractère exceptionnel de par l'originalité et la très grande pertinence sociale du sujet traité, tout en notant la richesse conceptuelle et empirique du travail qui a été mené. En ces temps de multiplication des conflits dans le monde, proposer un cadre conceptuel permettant des rapprochements entre l'éducation relative à l'environnement et l'éducation à la paix, définir les fondements et les pratiques de l'éducation relative à l'environnement qui renforceraient des processus et des «dynamiques de paix» et en préciser notamment les dimensions identitaires, constitue une avancée théorique importante.

5 Il faut préciser que cette thèse a pris corps grâce à une étude de cas réalisée au Liban auprès des acteurs de l'Organisation Non Gouvernementale libanaise IndyACT, à laquelle s'est ajoutée l'exploration complémentaire de deux initiatives dans le domaine spécifique de la consolidation environnementale de la paix : celle de l'organisation non gouvernementale « Community Gardens Association (CGA)» en Bosnie-Herzégovine, et celle de l'ONG « Friends of the Earth Middle East » (FOEME), qui œuvre en Jordanie, en Israël et en Palestine. Cette thèse qui allie ainsi une conceptualisation théorique de haut niveau à un travail empirique d'envergure témoigne de la détermination et de l'engagement authentique de son auteure dans ces questions d'éducation conjointe à la paix et à l'environnement.

6 Tel que Madame Naoufal le précise dans sa thèse : «Il semble que les fondements et les pratiques d'éducation qui s'inscriraient de manière féconde dans des programmes de consolidation environnementale de la paix sont de nature à participer à la construction d'une identité biorégionale ou environnementale, où l'appartenance et la responsabilité à l'égard d'un environnement partagé prennent le pas sur les affiliations identitaires d'ordre national, ethnique, religieux, confessionnel, etc. Ainsi, l'action collective, dialogique et réflexive autour de l'environnement partagé est fondamentale en cela qu'elle pourrait permettre de rallier les personnes et les communautés, au Liban comme dans d'autres contextes, et de favoriser une prise de conscience à la fois de la pluralité et de l'humanité partagée de nos identités. » Il s'agit donc de réfléchir ici aux fondements et aux manières d'éduquer à un rapport à soi, au monde et à l'autre repensé, vers plus de solidarité, d'authenticité, de partage et de respect des êtres et des lieux. Autant de considérations essentielles, en particulier dans des situations de conflits majeurs.

7 Le prix Lucie-Samson-Turcotte 2013 pour le doctorat a également été décerné à la thèse de doctorat de Étienne van Steenberghe : «Les représentations sociales des liens entre la santé et l'environnement. Vers des pratiques éducatives appropriées en matière de santé environnementale auprès de populations défavorisées en milieu urbain ».

8 Accordant une attention particulière aux questions écologiques et de santé dans le cas de populations appauvries en milieu urbain, et considérant qu'en dépit des diverses actions mises en œuvre par les politiques gouvernementales et les organisations communautaires, ces populations demeurent particulièrement vulnérables, la thèse primée porte un regard sur les liens entre la santé et l'environnement de proximité tel qu'il est exprimé par les personnes défavorisées en vue de contribuer au développement de stratégies d'intervention éducative en santé et en environnement. L'étude a été menée dans deux quartiers urbains: celui de Pointe-Saint-Charles à 
Montréal (Québec, Canada) et celui des Marolles à Bruxelles (Belgique), quartiers où l'on retrouve une concentration de personnes très défavorisées sur les plans social et économique. Les problèmes de santé liés à des conditions environnementales défavorables y sont particulièrement présents. La thèse permet de cerner les caractéristiques des deux quartiers à l'étude et d'étudier les rapports à la santé, à l'environnement et les liens entre les deux que proposent les personnes rencontrées, afin de faire émerger des pistes pour la poursuite de la recherche dans ce domaine et pour de meilleures stratégies d'intervention éducatives auprès de ces populations. Résolument ancrée dans une éducation relative à l'environnement sociocritique, il s'agit ici de donner véritablement la parole aux personnes les moins bien nanties afin de tenir compte de leurs préoccupations et de leurs représentations de leur environnement et de leur santé, pour ancrer des initiatives éducatives dans des dialogues plus authentiques, allant au-delà de la simple information où sensibilisation qui semblent peu porter fruit. Les membres du jury ont tenu à récompenser ce travail de grande qualité notamment pour sa très grande pertinence sociale et pour la position de son auteur qui met véritablement en scène la parole des sujets rencontrés.

Le prix Lucie-Samson-Turcotte 2013 pour un mémoire de maîtrise a pour sa part été remis à Madame Marie-Ève Marleau pour son mémoire intitulé « Les processus de prise de conscience et d'action environnementales: le cas d'un groupe d'enseignants en formation en éducation relative à l'environnement ».

10 Les problèmes actuels y sont déclinés dans leurs dimensions environnementales, socioéconomiques et politiques, culturelles et identitaires. Ce qui conduit à souligner l'importance de travailler la prise de conscience et l'engagement dans l'action en éducation relative à l'environnement et à proposer une première articulation de ces concepts sur le plan théorique.

11 Pour illustrer plus avant la qualité de ce mémoire, voici un extrait de l'évaluation faite par un des membres du jury:

12 «La première qualité de ce mémoire est sa richesse théorique qui témoigne d'une maitrise de la documentation consultée ainsi que d'une capacité à articuler divers concepts parfois éclairés par des champs différents. Les synthèses qui en résultent ont une excellente valeur scientifique. Il s'agit donc d'un mémoire de maîtrise de haut calibre. La consultation de sources documentaires en trois langues apporte une richesse particulière à ce projet et à l'étude de cas réalisée au Mexique. Sa pertinence scientifique, sociale et environnementale est grande. En effet, si être conscient des problématiques sans que cette conscientisation soit accompagnée d'un agir proenvironnemental est bien documenté sur le plan scientifique, le fossé qui sépare « la conscience environnementale » de « l'action environnementale » a été peu explorée. Or Madame Marleau propose des éléments d'arrimage entre ces deux concepts, sa contribution au champ de l'éducation relative à l'environnement est donc très importante.»

13 L'étude de cas contribue à préciser comment la conscience environnementale et l'action environnementale prennent un sens et s'actualisent en fonction d'un contexte scolaire, social et environnemental particulier, ici dans un contexte scolaire mexicain. La discussion générale qui en ressort précise quelles sont les principales approches et stratégies pédagogiques en éducation relative à l'environnement susceptibles de favoriser les processus de prise de conscience et d'action environnementales. 
L'ensemble est d'une grande cohérence et très bien écrit. Il s'agit donc d'un mémoire de maîtrise exemplaire à tout point de vue.

\section{BIBLIOGRAPHIE}

Nayla Naoufal (2012). La contribution de l'éducation relative à l'environnement à la construction d'une dynamique de paix. Doctorat en Sciences de l'environnement, Université du Québec à Montréal (UQAM) - https://archipel.uqam.ca/4953/

Étienne van Steenberghe (2012). Les représentations sociales des liens entre la santé et l'environnement. Vers des pratiques éducatives appropriées en matière de santé environnementale auprès de populations défavorisées en milieu urbain. Doctorat en Éducation et santé publique, Université du Québec à Montréal (UQAM) et Université catholique de Louvain (Belgique) - https://archipel.uqam.ca/ $5150 /$

Marie-Ève Marleau (2011). Les processus de prise de conscience et d'action environnementales : le cas d'un groupe d'enseignants en formation en éducation relative à l'environnement. Maîtrise en Éducation. Université du Québec à Montréal (UQAM) - https://archipel.uqam.ca/4234/ 